\title{
CALIDAD DEL CUIDADO DE ENFERMERÍA AL RECIEN NACIDO EN ALOJAMIENTO CONJUNTO, HOSPITAL SANTA MARÍA DEL SOCORRO, ICA-2017
}

Quality of nursing care for the newborn in joint accommodation, Hospital Santa María of Socorro, Ica-2017

Dra. Zonia Felicitas Quispe Quispe, $2, a, b$, Nut. Yolanda Chinarro de Pun ${ }^{1, d}$, Lic. Enf. Ana Cecilia Flores García $^{2, a, c}$, Lic. Enf. Patricia Bonifaz Flores $2, a$

${ }^{1}$ Facultad de Enfermería de la Universidad Nacional San Luis Gonzaga de Ica

${ }^{2}$ Hospital Santa María del Socorro de Ica.

a Licenciada en Enfermería, ${ }^{b}$ Doctor en Salud Pública, 'Magíster en Educacion, ${ }^{d}$ Nutricionista

\section{RESUMEN}

Objetivo: Analizar la calidad del cuidado de enfermería al recién nacido en alojamiento en conjunto, en el Hospital Santa María del Socorro de Ica, en el año 2017. Materiales y Métodos: Estudio no experimental, descriptivo, transversal, muestra de 120 binomios en alojamiento conjunto. Resultados: En forma global la calidad del cuidado de enfermería es buena. El 78,3\% de las madres perciben calidad del cuidado de enfermería buena, 12,5\% excelente, y 9,2\% mala calidad. En la dimensión estructura, 78,3\% perciben calidad buena, 12,5\% mala, y 9,2\% excelente. En la dimensión proceso, 60\% perciben calidad buena, 21,7\% mala, y 18,3\% excelente. En la dimensión resultado, 70\%, perciben la calidad buena, 19,2\% excelente, y 10,8\% señalan mala calidad. Las características sociodemográficas predominantes fueron: Edad 21 a 25 años (35\%), instrucción superior (62,5\%), número de hijos: 1 hijo (56,7\%), estado civil conviviente $(50,8 \%)$, ocupación estudiante (33,3\%), e ingreso económico S/860 a 1200 soles $(35,8 \%)$. Conclusiones: En forma global, así como en sus dimensiones la calidad del cuidado de enfermería es buena.

Palabras clave: Calidad, cuidado de enfermería, recién nacidos, alojamiento conjunto.

\section{SUMMARY}

Objective: To analyze the quality of nursing care to the newborn in a joint accommodation, at the Hospital Santa María of Socorro Ica, in the year 2017. Materials and Methods: A nonexperimental, descriptive, cross-sectional study of 120 binomials in housing set. Results: Overall the quality of nursing care is good. $78.3 \%$ of mothers perceive quality of good nursing care, $12.5 \%$ excellent, and $9.2 \%$ poor quality. In the structure dimension, $78.3 \%$ perceived good quality, $12.5 \%$ bad, and $9.2 \%$ excellent. In the process dimension, $60 \%$ perceive good quality, $21.7 \%$ bad, and $18.3 \%$ excellent. In the result dimension, $70 \%$ perceive good quality, $19.2 \%$ excellent, and $10.8 \%$ indicate poor quality. The predominant sociodemographic characteristics were: Age 21 to 25 years $(35 \%)$, higher education $(62.5 \%)$, number of children: 1 child $(56.7 \%)$, civil status of the partner (50.8\%), student occupation (33.3\%), and Economic income S / 860 to 1200 soles (35.8\%). Conclusions: Overall, as well as in its dimensions, the quality of nursing care is good. Key words: Quality, nursing care, newborns, joint accommodation.

\section{INTRODUCCIÓN}

La protección de la vida y la salud de los recién nacidos es una de las prioridades del Estado, así como cautelar el derecho a la atención integral en salud con calidad y oportunidad, desde el nacimiento; las estadísticas indican que durante el año 2015 , el $69,3 \%$ de los nacidos vivos fueron atendidos con el SIS, $19,5 \%$ en el Seguro Social de Salud (EsSalud), 2,9\% con prestadores privados, y que $7,6 \%$ no cuentan con un seguro de salud, en 
menores proporciones, $1 \%$ fueron atendidos en la Sanidad de las Fuerzas Armadas (FFAA) , $0,3 \%$ en la sanidad Policial, y $0,4 \%$ en otros prestadores de salud (1).

El alojamiento en conjunto, es una de las áreas más importantes para el fortalecimiento del binomio madre - hijo, en donde el profesional de enfermería provee cuidados específicos para una buena interacción entre ambos y asimismo la realización de valoración de la salud del bebé.

En esta etapa, la madre necesita de cuidados específicos para el bebé por lo que el profesional de enfermería debe estar capacitado y tener conocimientos, habilidades y actitudes para atender a todas las necesidades tanto fisiológicas como afectivas para el recién nacido y la educación de la madre para el cuidado del bebé. Siendo necesario que este cuidado de enfermería se ejecute eficazmente logrando así que la salud materna sea adecuada.

Según el Instituto Nacional de Estadística e Informática (INEI), al año 2015 se reportaron 558 mil 466 nacimientos. Por día se inscriben 1530 recién nacidos, que representa 64 niñas y niños registrados por cada hora. Sabemos por estudios diversos que en el periodo neonatal se da la mayor morbilidad en la población infantil por su carácter de transición entre la vida intrauterina y extrauterina (2), en esta etapa se requiere de una mayor atención de Enfermería, un cuidado de calidad para garantizar la vida y la salud del recién nacido. La Calidad de la Atención de Enfermería se puede definir como "la consecución del conjunto de características y acciones que posibilitan la restauración en cada paciente, del nivel de salud que nos es dado remitirle" (3).

Donabedian agrupa en tres categorías los componentes fundamentales del servicio, susceptibles de generar satisfacción: componente técnico, componente interpersonal, y componente del entorno y sus comodidades. Conocer la satisfacción del paciente puede permitir predecir, entre otras cosas, el cumplimiento terapéutico y el retorno al mismo centro frente a otro episodio. En consecuencia, la satisfacción representa la vivencia subjetiva derivada del cumplimiento o incumplimiento de las expectativas que tiene un sujeto con respecto a algo (4).

Por otra parte, el cuidado se ha definido como el conjunto de categorías que involucran la comunicación verbal y no verbal, la minimización del dolor físico, la empatía para atender el todo, y el envolvimiento, que se refiere a la aproximación entre el cuidador y el ser cuidado como finalidad terapéutica (5).

El alojamiento conjunto, también llamado habitación compartida, consiste en brindar la atención médica, enfermería y del personal de trabajo social a la madre y al recién nacido de manera tal que se favorezca su contacto temprano piel a piel, desde el momento del nacimiento y durante todo el tiempo que permanecen en el hospital, con motivo del parto. Para ello, madre e hijo son alojados conjuntamente, con objeto de fortalecer la relación afectiva entre ellos y de lograr que el niño inicie con la alimentación al seno materno y se continúe a libre demanda; asimismo, esto permite brindar a la madre información práctica acerca de la alimentación y cuidados de su hijo (6).

El cuidado del recién nacido normal tiene como objetivo supervisar que el proceso de adaptación del recién nacido se realice en forma normal y ayudar a la madre a comprender las características propias de este período y de fenómenos fisiológicos que no ocurren en ninguna otra edad. A los padres, en especial a la madre les compete el cuidado de su hijo recién nacido. Hay un período de adaptación clave en los primeros días entre la madre y el recién nacido que abarca funciones biológicas, psicológicas y espirituales (7).

La calidad es un atributo que distingue a un producto o servicio, en los niveles actuales de competencia y exigencia global, los 
usuarios solicitan servicios de calidad, especialmente cuando se trata de la salud. La forma más usual y adecuada de establecer la calidad de un servicio se da a través de la percepción de los usuarios quienes manifiestan su satisfacción con las dimensiones del servicio como el buen tato, la comunicación, el respeto, la continuidad y la confortabilidad.

La calidad en la atención de enfermería comprende dos dimensiones: La dimensión técnica, que requiere de la aplicación de la ciencia la destreza y la tecnología, y la dimensión interpersonal que incluye valores, respeto; de tal manera que con ambas se logre los máximos beneficios para la salud. Una tercera dimensión que contribuye es la estructura llamada también infraestructura, es decir las condiciones físicas, iluminación limpieza, ventilación y el ambiente.

En el servicio de maternidad del Hospital Santa María del Socorro ( HSMS) en la práctica del día a día se observan algunos problemas que no permiten dar una atención de enfermería de calidad, como por ejemplo: no existe una atención personalizada en el apoyo emocional hacia la puérpera, debido al número de binomios que en promedio son 18 por cada día, el abastecimiento de medicamentos que falta en algunas ocasiones, el incremento cada vez mayor de usuarias del servicio de maternidad, de diferente condición socioeconómica y cultural, la falta de capacitación enfocada a la atención del usuario, la atención al binomio que se comparte entre enfermería y obstetricia, y el clima laboral, entre otros.

La importancia de la investigación radica entonces en conocer las percepciones de las madres sobre la calidad del cuidado de enfermería que reciben los recién nacidos en alojamiento conjunto durante el periodo de puerperio, en el Hospital Santa María del Socorro de Ica durante el año 2017, con esta información se harán propuestas para una mejor asistencia de los neonatos y sus madres en puerperio.

\section{MATERIAL Y MÉTODOS.}

El estudio efectuado ha tenido en cuenta, los principios éticos de autonomía, beneficencia y justicia, siendo el tipo de investigación descriptivo, no experimental, de corte transversal, ejecutándose en madres que se encuentran en alojamiento conjunto en el servicio de maternidad del Hospital Santa María del Socorro de Ica, durante los meses de enero a febrero del 2017.

La población lo constituyeron las madres o puérperas en alojamiento conjunto del servicio de maternidad del Hospital santa María del Socorro de Ica, durante los meses de enero a febrero del 2017. Como muestra se obtuvo 120 binomios en alojamiento conjunto que fueron seleccionados en forma intencional.

Se utilizaron los métodos y técnicas: Inductivo, aplicando las encuestas; el método deductivo para interpretar los resultados; y el método matemático para procesar la información utilizando fórmulas.

Se utilizó como técnicas: La encuesta, la observación, el análisis y la síntesis. La información que se requiere para llevar a cabo la investigación fue recogida de fuentes directas e indirectas

Utilizamos el instrumento de María Augusta Ruales y Nathaly Ortiz (2015) (8) basado en la teoría y modelo de Donabedian que tiene 21 reactivos, 7 de la dimensión Estructura, 7 de Proceso y 7 de Resultado, en escala Likert de 4 niveles: Nunca, A veces, Frecuentemente, y Siempre, de 1 a 4 puntos respectivamente. Con un puntaje máximo de 28 puntos para cada dimensión, y mínimo de 7 puntos.

La validación del cuestionario se realizó mediante criterio de 3 expertos y la confiabilidad se determinó mediante Alfa de Cronbach en el modo de test y retest, con una muestra de 10 puérperas.

Inicialmente se hizo un análisis descriptivo, de frecuencias de puntajes directos, luego 
se hizo un análisis de la variable calidad del cuidado de enfermería a neonatos en alojamiento conjunto. Se utilizó la estadística descriptiva y se hizo un análisis 95\% de confianza, con el paquete estadístico SPSS versión 21,0 .

\section{RESULTADOS.}

En cuanto a los datos sociodemográficos, se observa que el $23,3 \%$ de las madres de los recién nacidos en alojamiento conjunto tienen entre 16 a 20 años, 30\% afirman tener 21 a 25 años, $24,2 \%$ entre 26 a 30 años, $10 \%$ entre 31 a 35 años, y 7,5\% tienen de 36 a 40 años de edad. El 2,5\% de las madres de los recién nacidos en alojamiento conjunto tienen solo instrucción primaria, $35 \%$ educación secundaria y $62,5 \%$ tienen instrucción superior. El $56,7 \%$ de las madres de los recién nacidos en alojamiento conjunto tienen un hijo, 25,8\% tienen 2 hijos, $15 \%$ tienen 3 hijos, y 2,5\% de las madres tienen 4 hijos. El 22,5\% de las madres de los recién nacidos en alojamiento conjunto son solteras, $26,7 \%$ casadas, y $50,8 \%$ son convivientes. El 33,3\% de las madres de los recién nacidos en alojamiento conjunto son estudiantes, $11,7 \%$ son amas de casa, $29,2 \%$ tienen trabajo dependiente (secretarias, profesoras, técnicos de enfermería), y el $25,8 \%$ tienen trabajo independiente (cosmetólogas, mototaxistas, comerciantes, entre otros). El $24,2 \%$ de las madres de los recién nacidos en alojamiento conjunto tienen un ingreso económico mensual de 600 a 850 soles, $35,8 \%$ entre 860 a 1200 soles, $21,7 \%$ de 1250 a 1500 soles, y $18,3 \%$ tienen ingresos de 1600 soles a más.

En cuanto a la calidad de cuidado de enfermería, el 78,3\% de las madres de los recién nacidos en alojamiento conjunto, perciben que la calidad del cuidado de enfermería es buena, el $12,5 \%$ señala que es excelente, y $9,2 \%$ perciben que es mala la calidad del cuidado de enfermería (Tabla 1).
Por dimensiones de la calidad del cuidado, se observa que el $78,3 \%$ de las madres de los recién nacidos en alojamiento conjunto, perciben a la dimensión "estructura" de la calidad del cuidado de enfermería, como buena, el $12,5 \%$ mala, y $9,2 \%$ excelente calidad. El $60 \%$ de las madres de los recién nacidos en alojamiento conjunto, perciben a la dimensión "proceso" de la calidad del cuidado de enfermería, como buena, el $21,7 \%$ malo, y $18,3 \%$ excelente. El $70 \%$ de las madres de los recién nacidos en alojamiento conjunto, perciben a la dimensión "Resultado" como buena, el 19,2\% excelente, y 10,8\% malo (Tabla 2.)

Tabla 1. Calidad del cuidado de enfermería al recién nacido, Servicio de Maternidad del Hospital Santa María del Socorro, Ica 2017

\begin{tabular}{lcc}
\hline $\begin{array}{l}\text { Calidad del cuidado } \\
\text { al recién nacido }\end{array}$ & $\mathbf{N}^{\circ}$ & $\%$ \\
\hline Excelente & 15 & 12,5 \\
Buena & 94 & 78,3 \\
Mala & 11 & 9,2 \\
\hline Total & 120 & $100 \%$ \\
\hline
\end{tabular}

Fuente: Encuesta aplicada a las madres de los recién nacidos

Tabla 2. Dimensiones de la calidad del cuidado de enfermería al recién nacido, Servicio de Maternidad del Hospital Santa María del Socorro, Ica 2017

\begin{tabular}{lcc}
\hline \multicolumn{3}{c}{ Calidad del cuidado al recién nacido } \\
\hline Dimensión Estructura & $\mathbf{N}^{\circ}$ & $\%$ \\
\hline Excelente & 15 & 12,5 \\
Buena & 94 & 78,3 \\
Mala & 11 & 9,2 \\
\hline Total & 120 & $100 \%$ \\
\hline Dimensión: Proceso & $\mathbf{N}^{\circ}$ & $\%$ \\
\hline Excelente & 22 & 18,3 \\
Buena & 72 & 60,0 \\
Mala & 26 & 21,7 \\
\hline Total & 120 & $100 \%$ \\
\hline Dimensión: Resultado & $\mathbf{N}^{\circ}$ & $\%$ \\
\hline Excelente & 23 & 19,2 \\
Buena & 84 & 70,0 \\
Mala & 13 & 10,8 \\
\hline Total & 120 & $100 \%$ \\
\hline
\end{tabular}

Fuente: Encuesta aplicada a las madres de los recién nacidos 


\section{DISCUSIÓN}

La percepción de las madres sobre el cuidado al recién nacido por parte de la enfermera, constituye un elemento clave para determinar la eficiencia de la atención en el binomio madre-hijo, condicionado a una buena calidad en todos los procedimientos que realiza la enfermera durante el periodo de puerperio, logrando así una mejor calidad de vida tanto para la madre como para el recién nacido.

En el estudio se analizó cómo es la calidad del cuidado de enfermería al recién nacido en alojamiento conjunto del servicio de maternidad del Hospital Santa María del Socorro de Ica, encontrándose en los datos sociodemográficos que existen en mayor proporción madres con edades comprendidas entre los 21 a 25 años (35\%), seguido de las edades de 26 a 30 años $(24,2 \%)$. Asimismo, se encontró que el nivel instrucción predominante fue el superior $(62,5 \%)$ seguido de la educación secundaria (35\%). En cuanto al número de hijos en las madres, se encontró que la mayoría tiene 1 solo hijo (56,7\%), seguido de 2 hijos (25,8\%). En el estado civil de las madres predomina la convivencia $(50,8 \%)$, seguido del estar casada (26,7\%); y en la ocupación de las madres predomina el ser estudiante $(33,3 \%)$, seguido del trabajo dependiente (secretarias, profesoras, etc) con $29,2 \%$. Finalmente se analizó el ingreso económico de las madres en estudio, encontrando que mayormente tienen ingresos de $\mathrm{S} / 860$ a 1200 soles, seguido de $S / 600$ a 850 soles.

La calidad del cuidado fue analizada mediante un instrumento basado en la teoría de Donabedian con 21 reactivos, en donde las madres de los recién nacidos reflejaron su percepción sobre el cuidado que recibieron por parte del profesional de enfermería, encontrándose en forma global que el cuidado fue mayormente bueno con $78,3 \%$, seguido de excelente $12,5 \%$, y malo con $9,2 \%$. Al realizar la comprobación de hipótesis mediante la prueba de $t$ de student, dio como resultado $=103,580, p, 000$ el cual nos indicó alta significación estadística, comprobando que el cuidado en forma global es bueno. Resultados que podemos compararlos con el estudio de Blanco J; Jiménez B. y Nieto B. (2010) (9) quienes en sus resultados encontraron un alto nivel de satisfacción con 84,7\%. Asimismo, en el estudio de Ruales M; y Ortiz N. (2015) (8) hallaron en sus resultados que la calidad de atención al neonato fue bueno-excelente. Sailema S. (2014) (10) encontró que la calidad de cuidado de enfermería en el recién nacido, mayormente es buena $(70 \%)$. En el estudio de Delgadillo D. (2014) (11) también encontró resultados favorables con satisfacción media a alta. Sin embargo, el estudio de Paiva D. et al. (2014) (12) difiere con nuestro estudio, al encontrar en sus resultados que la calidad de atención en el puerperio aún es limitada.

El análisis de la calidad del cuidado de enfermería, también fue analizado según sus 3 dimensiones, los cuales nos dan a conocer diversas percepciones sobre cada una de las intervenciones que realiza el profesional de enfermería. En la dimensión "estructura" las madres de los recién nacidos percibieron mayormente el cuidado de enfermería como bueno $78,3 \%$, cuidado malo $12,5 \%$, y cuidado excelente con $9,2 \%$. Esta dimensión estuvo relacionada a: medicamentos disponibles, si la enfermera cambia la ropa de cuna o incubadora según necesidad, si el personal de enfermería es suficiente, si la comodidad hospitalaria es buena, si la temperatura, ventilación e iluminación es adecuada, y si los alimentos son adecuados.

En la segunda dimensión "proceso" las madres de los recién nacidos percibieron mayormente la calidad del cuidado como buena $69 \%$, seguido de calidad mala $21,7 \%$, y excelente con 18,3\%. Esta dimensión estuvo referida a: $\mathrm{Si}$ el personal de enfermería le llama por su nombre, si los procedimientos son adecuados, si le ofrecen las condiciones necesarias de intimidad, si le brindan apoyo emocional, si se interesan porque su estancia sea agradable, y si le 
enseñan sobre los cuidados que debe realizar al bebé.

Finalmente, en la dimensión "resultado", las madres percibieron mayoritariamente la calidad de cuidado como buena $70 \%$, excelente $19,2 \%$, y calidad mala 10,8\%. Esta dimensión estuvo relacionada a: Si existe continuidad en los cuidados de enfermería, si les responden a sus preguntas, si satisfacen las necesidades de higiene, eliminación y descanso, si durante la hospitalización el bebé ha tenido complicaciones en salud, si le hacen sentirse segura en la atención al bebé, si el bebé tuvo cuidados en el momento requerido, y si recomendaría el cuidado de enfermería recibido.

\section{CONCLUSIONES}

1. La calidad del cuidado de enfermería en recién nacidos es $78,3 \%$ buena, $12,5 \%$ excelente, y $9,2 \%$ mala.

2. En la dimensión estructura, 78,3\% perciben buena calidad del cuidado, $12,5 \%$ mala, y $9,2 \%$ excelente.

3. En la dimensión proceso, $60 \%$ perciben buena calidad del cuidado, $21,7 \%$ mala, y $18,3 \%$ excelente.

4. En la dimensión resultado, el $70 \%$ perciben buena calidad del cuidado, $19,2 \%$ excelente, y $10,8 \%$ mala.

\section{RECOMENDACIONES}

1. La Jefatura de enfermería, debe mejorar la disponibilidad de medicamentos, y sensibilizar a las enfermeras para que se dirijan a los pacientes por su nombre, se interesen por una estancia agradable, y que la atención sea oportuna.

2. La Directora de Calidad del Hospital debe elaborar indicadores de calidad de servicio, y llevar a cabo por lo menos 2 veces al año encuestas de satisfacción.

3. Otros investigadores deben continuar la investigación, asociando la variable principal con otras variables.

\section{LIMITACIONES DEL ESTUDIO}

El estudio no tuvo limitaciones, desarrollándose exclusivamente con madres de recién nacidos en alojamiento conjunto, contándose con presupuesto, metodología y tiempo de ejecución en forma apropiada.

\section{Correspondencia:}

Dra. Zonia Quispe Quispe

Correo electrónico:

zoniaquispe@hotmail.com

Celular: +51956004215

\section{REFERENCIAS BIBLIOGRÁFICAS}

1. Instituto Nacional de Estadística e Informática. Nota informativa: Al 30 de Junio de 2015 el Perú tiene 31 millones 151 mil 643 habitantes. Lima, Perú 2015. Disponible en:

https://www.inei.gob.pe/media/MenuRe cursivo/noticias/nota-de-prensa-n0992015-inei.pdf

2. Ministerio de Salud. Boletín estadístico de nacimientos Perú: 2015. Sistema de Registro del Certificado de Nacido vivo en línea. Lima, Perú. 2016.

3. Moreno R. La calidad de la acción de Enfermería. Enfermería Global. 2005.

4. Massip, C. La evaluación de la satisfacción en salud: un reto a la calidad. Rev Cubana de salud Pública, 1-10. 2008.

5. Baggio M. Significado de cuidado para profissionais da equipe de enfermagem. Rev Electrónica de Enfermería 2006; 8 (1):9-16.

6. Ministerio de Sanidad, Política social e Igualdad. IHAN. Calidad en la asistencia profesional al nacimiento y la lactancia. Gobierno de España. Madrid, España; 2011. 
7. Landeros E, Salazar T. Reflexiones sobre el cuidado. Rev. Enferm. Inst Mex Seguro. 2006; 14 (3): 121-122.

8. Ruales M, Ortiz N. La calidad de atención de enfermería relacionada con el grado de satisfacción de las madres de los neonatos del Hospital Carlos Andrade Marín. Tesis Pregrado. Universidad Central del Ecuador. Facultad de Ciencias Médicas. Quito, Ecuador. 2015r. (Internet) Disponible en:

http://www.dspace.uce.edu.ec/bitstrea $\underline{\mathrm{m} / 25000 / 4782 / 1 / T-U C E-0006-137 . p d f}$

9. Blanco J, Jiménez $B$, Nieto $B$. Satisfacción de usuarias sobre la atención brindada por enfermería en alojamiento conjunto del hospital del niño y la mujer. Tesis pregrado. Universidad Autónoma de San Luis Potosí. México. Facultad de Enfermería. 2010. (Internet) Disponible en: http://ninive.uaslp.mx/ispui/ bitstream/i/3004/4/LEN1SUS01001.pdf

10. Sailema S. Intervenciones que cumplen las enfermeras/os en el manejo del recién nacido y el grado de satisfacción de las madres en alojamiento conjunto, en el servicio de maternidad del Hospital Provincial General de Latacunga. Tesis Pregrado. Universidad técnica de Ambato. Enfermería. Ambato, Ecuador. 2014. (Internet) Disponible en: http://repositorio.uta.edu.ec/bitstream/ 123456789/7969/1/Sailema\%200rtiz,\% 20Silvia\%20Elizabeth.pdf
11. Delgadillo D. Satisfacción de la madre del recién nacido hospitalizado sobre la calidad del cuidado del profesional de enfermería en el Servicio de Neonatología del Instituto Nacional Materno Perinatal. Tesis especialidad. Lima, Perú. Facultad de Medicina, Universidad Nacional Mayor de San Marcos; 2014. (Internet) Disponible en:

http://ateneo.unmsm.edu.pe/ateneo/bits tream/123456789/4309/1/Delgadillo M orales Doris 2014.pdf

12. Paiva D. et al. Cuidados al binomio madre-hijo en el puerperio inmediato: Estudio descriptivo. Universidad Federal Fluminenses. Escuela de Enfermería Aurora de Alfonso Costa. Brasil, 2014. (Internet) Disponible en: http://www.objnursing.uff.br/index.php/n ursing/article/view/4231

Recibido: 11/12/2020

Aprobado para Publicación:03/03/2021 